\title{
Resistencia antimicrobiana y nuevos principios bioactivos
}

\section{Antimicrobial resistance and novel bioactive principles}

J. Selva Andina Res. Soc. 2019;10(1):1-3.

La resistencia a los antimicrobianos es una amenaza latente para la salud humana y el desarrollo económico, e inclusive a través de procesos evolutivos naturales, han hecho posible que los microorganismos sean resistentes a estos, asimismo hoy en día, algunas infecciones bacterianas son intratables con los antimicrobianos existentes. ${ }^{1}$

Actualmente se registra presencia de multidrogo-resistentes, tal como Staphylococcus aureus meticilino resistente $(\mathrm{SAMR})^{2}$ además a través de datos de vigilancia de infecciones, se estima una alta prevalencia de Enterobacterias resistentes a cefalosporinas de tercera generación (3GCREB) siendo aproximadamente 16 veces mayor que la indicada por la incidencia de infección de ingreso ${ }^{3}$ e incluso una preocupación por la resistencia a otro grupo de antibióticos $\beta$-lactámicos como los carbapenémicos. ${ }^{4}$

Frente a esta realidad ha surgido el interés por la búsqueda de compuestos bioactivos o nuevos fármacos a partir de extractos de plantas medicinales, así como también conocer su perfil bioquímico y evaluar su potencial antimicrobiano ${ }^{5}$ siendo de interés algunas familias tales como Solanaceae, Myristicaceae, Lamiaceae, Asteraceae y en otras familias, que se ha evidenciado su capacidad de inhibir el crecimiento de bacterias como Bacillus subtilis, Staphylococcus aureus, Escherichia coli, Pseudomonas aeruginosa y hongos como Candida albicans, Trichophyton mentagrophytes, Microsporum gypseum y Sporothrix schenckii. ${ }^{6}$

Además a través del veneno de algunos artrópodos, como Apis mellifera se ha comprobado su potencial actividad antibacteriana contra diferentes cepas bacterianas, siendo de interés conocer su naturaleza química de estos principios activos e inclusive su mecanismo de acción frente estos microorganismos. ${ }^{7}$ Asimismo veneno de escorpión se tiene mayor evidencia científica de su actividad, inclusive con el veneno crudo de la especie Heterometrus xanthopus ${ }^{8}$ como también de sus compuestos purificados en el caso del veneno de escorpiones mexicanos de las especies Hadrurus aztecus y Vaejovis mexicanus con péptidos denominados hadrurina y vejovina con capacidad antibacteriana frente a grampositivas y gramnegativas. ${ }^{9}, 10$

No solo se ha señalado que el veneno de algunos artrópodos (insectos o arácnidos) puede ser efectivo, sino que, también el veneno de serpientes de la familia Elapidae y Viperidae que han sido estudiadas por ser altamente tóxicas, aislándose componentes específicos en los venenos, como la fosfolipasa A2 y Laminoácido oxidasas, que también han demostrado ser efectivos, la familia Colubridae se tiene muy pocos estudios por el motivo de ser menos tóxicas, a pesar de que se han completado numerosos estudios sobre venenos de serpientes los resultados indican que sus componentes aislados son agentes antimicrobianos poderosos, pero varían en eficacia hacia diferentes clases de microbios. ${ }^{11}$

Nuestra biodiversidad ofrece alternativas novedosas frente a estos cambios que surgen ante una amenaza post- 
antibiótica, que pueden ser aplicadas con apoyo de estudios clínicos que ayudarían en a la terapia antimicrobiana, además deban sumarse la investigación básica y aplicada e incluso el empleo de tecnologías de vanguardia, que puedan dilucidar desde su estructura química, mecanismos de acción y entre otros.

\section{Literatura citada}

1. World Health Organization, Food and Agriculture Organization of the United Nations and World Organisation for Animal Health. Monitoring Global Progress On Addressing Antimicrobial Resistance (AMR) [Internet]. 2018 [Cited 2019 Jan 07]. Recuperado a partir de: https://www.who.int/ antimicrobial-resistance/publications/Analysis-report-of-AMR-country-se/en/

2. Ravensbergen SJ, Louka C, Ott A, Rossen JW, Cornish D, Pournaras S, et al. Proportion of asylum seekers carrying multi-drug resistant microorganisms is persistently increased after arrival in the Netherlands. Antimicrob Resist Infec Control 2019;8(6):1-7. DOI: https://doi.org/10.1186/s13756018-0455-5

3. Rohde AM, Zweigner J, Wiese Posselt M, Schwab F, Behnke M, Kola A, et al. Incidence of infections due to third generation cephalosporin-resistant Enterobacteriaceae- a prospective multicentre cohort study in six German university hospitals. Antimicrob Resist Infect Control 2018;27(159):1-7 Disponible en: https://doi.org/10.1186/s13756-018-0452-8

4. Zhang D, Cui K, Lu W, Bai H, Zhai Y, Hu S, et al. Evaluation of carbapenem use in a tertiary hospital: antimicrobial stewardship urgently needed. Antimicrob Resist Infec Control 2019;8(5):1-7. DOI: https://doi.org/10.1186/s13756-018-0449-3

5. Mustafa G, Arif R, Atta A, Sharif S, Jamil A. Bioactive compounds from medicinal plants and their importance in drug discovery in Pakistan. Matrix Sci Pharm 2017;1(1):17-26. Recuperado a partir de: DOI: http://www.doi.org/10.26480/msp.01.2017.17.26

6. Rojas R, Bustamante B, Bauer J, Fernández I, Albán J, Lock O. Antimicrobial activity of selected Peruvian medicinal plants. J Ethnopharmacol 2003;88(2-3):199-204. DOI: https://doi.org/10.1016/ $\underline{\mathrm{S} 0378-8741(03) 00212-5}$

7. Hegazil AG, EL Feel MA, Abdel Rahman EH, Al Fattah MA. Antibacterial activity of bee venom collected from Apis mellifera carniolan pure and hybrid races by two collection methods. Int J Curr Microbiol App Sci 2015;4(4):141-9.

8. Ahmed U, Mujaddad-ur-Rehman M, Khalid N, Fawad SA, Fatima A. Antibacterial activity of the venom of Heterometrus xanthopus. Indian J Pharmacol 2012;44(4):509-11. DOI: https://doi.org/ 10.4103/0253-7613.99332

9. Torres Larios A, Gurrola GB, Zamudio FZ, Possani LD. Hadrurin, a new antimicrobial peptide from the venom of the scorpion Hadrurus aztecus. Eur J Biochem 2000;267(16):5023-31. DOI: https://doi.org/10.1046/j.1432-1327.2000.01556.x

10. Hernández Aponte CA, Silva Sanchéz J, Quintero Hernandez V, Rodríguez Romero A, Balderas C, Possani LD, et al. Vejovine, a new antibiotic from the scorpion venom of Vaejovis mexicanus. Toxicon 2011;57(1):84-92. DOI: https://doi.org/10.1016/j.toxicon.2010.10.008 
11. Charvat RA, Strobel RM, Pasternak MA, Klass SM, Rheubert JL. Analysis of snake venom composition and antimicrobial activity. Toxicon 2018;150:151-67. DOI: https://doi.org/10.1016/ j.toxicon.2018.05.016

Orlando Pérez-Delgado Laboratorio de Microbiología Facultad de Ciencias de la Salud Universidad Señor de Sipán Chiclayo - Perú Dirección postal: 14001 Tel: +51074481610 E-mail address: operezd $@$ gmail.com 